\section{A new page on the road book of inorganic mercury in fish body - tissue distribution and elimination following waterborne exposure and post-exposure periods}

\author{
Patrícia Pereira, ${ }^{\text {abcd }}$ Joana Raimundo, ${ }^{\mathrm{b}}$ Marisa Barata, ${ }^{\mathrm{e}}$ Olinda Araújo, ${ }^{\mathrm{b}}$ \\ Pedro Pousão-Ferreira, ${ }^{e}$ João Canário, ${ }^{\dagger}$ Armando Almeida ${ }^{c d}$ and Mário Pacheco ${ }^{a}$
}

Received 10th November 2014, Accepted 27th January 2015

DOI: 10.1039/c4mt00291a

www.rsc.org/metallomics

\begin{abstract}
There are several aspects of inorganic mercury (iHg) toxicokinetics in fish that remain undeveloped despite its environmental ubiquity, bioaccumulation capacity and toxicity. Thus, this study presents new information on the uptake, distribution and accumulation of $\mathrm{iHg}$ following water contamination by adopting a novel set of body compartments (gills, eye wall, lens, blood, liver, brain and bile) of the white sea bream (Diplodus sargus) over 14 days of exposure. Realistic levels of $\mathrm{iHg}$ in water $\left(2 \mu \mathrm{g} \mathrm{L}^{-1}\right)$ were adopted in order to engender reliable conclusions in the assessment of fish health. A depuration phase of 28 days was also considered with the purpose of clarifying iHg elimination. It was found that $\mathrm{iHg}$ was accumulated faster in the gills (within 1 day), which also had the highest accumulated levels among all the target tissues/organs. Moreover, iHg increased gradually with exposure time in all the tissues/organs, except for the lens that showed relatively unaltered levels throughout the experiment. After 14 days of exposure, lower values of $\mathrm{Hg}$ were recorded in the brain/ eye wall compared to in the liver, which is probably related with the presence of blood-organ protection barriers, which limit $\mathrm{iHg}$ influx. $\mathrm{iHg}$ reached the brain earlier than the eye wall ( 3 and 7 days, respectively) and, hence, higher accumulated levels were recorded in the former. A depuration period of 28 days did not allow for the total elimination of $\mathrm{iHg}$ in any of the tissues/organs. Despite this, $\mathrm{iHg}$ was substantially eliminated in the gills, blood and liver through two temporal phases, whereas the brain and eye wall were not able to eliminate $\mathrm{iHg}$ within this timeframe. The brain and eye wall are more "refractory" structures with regard to $\mathrm{iHg}$ elimination, and this could represent a risk for wild fish populations.
\end{abstract}

\section{Introduction}

Mercury ( $\mathrm{Hg}$ ) compounds have long triggered major concerns in terms of environmental and human health. This trace element is present in the aquatic environment in organic (mainly methylmercury: $\mathrm{MeHg}$ ) and inorganic forms (iHg), and both can be bioaccumulated by fish, inducing toxic effects. There are several studies addressing $\mathrm{Hg}$ kinetics in fish bodies e.g. ref. 1-4. Most of them are focused on the widely explored tissues, such as the liver, kidney, gills and muscles, while other potential target organs (e.g. the brain and neurosensory structures) have so far been neglected. The lack of

${ }^{a}$ Department of Biology and CESAM, University of Aveiro, 3810-193 Aveiro, Portugal

${ }^{b}$ IPMA - Portuguese Institute for the Sea and Atmosphere, Av. Brasilia,

50 1449-006 Lisbon, Portugal. E-mail: ppereira@ipma.pt; Fax: +351 21 3015948; Tel: +351213027172

${ }^{c}$ Life and Health Sciences Research Institute (ICVS), School of Health Sciences, University of Minho, Campus de Gualtar, 4710-057 Braga, Portugal

${ }^{e}$ IPMA - Aquaculture Research Station, 8700-005 Olhão, Portugal

${ }^{f}$ Centro de Química Estrutural, Instituto Superior Técnico, Universidade de Lisboa, Av. Rovisco Pais, 1049-001 Lisboa, Portugal

research on $\mathrm{Hg}$ accumulation and pathways in fish brain and eyes is an intriguing aspect in ecotoxicology, especially when considering their crucial roles in fish fitness and survival. Both the brain and eyes are protected by epithelial barriers (the blood-brain barrier [BBB] and the blood-retinal barrier [BRB]) that strictly regulate the selective transport of molecules from the bloodstream. ${ }^{5}$ BBB and BRB are highly restrictive membranes, but both can be crossed by essential elements such as $\mathrm{Mn}$ and $\mathrm{Fe}^{6}{ }^{6}$ In regard to $\mathrm{Hg}$ in fish, it is well established that the $\mathrm{Hg}$ may also reach the brain and eyes., However, the permeability of these barriers to $\mathrm{Hg}$ needs to be clarified in fish, in particular, whether it could be crossed bi-directionally, as well as the extent of $\mathrm{Hg}$ influx and efflux. The balance (or unbalance) between efflux and influx will inevitably lead to an accumulation, as reported for Fe in rodents' brains. ${ }^{8}$ Furthermore, fish lens has no direct blood supply, but has been seen to accumulate high levels of $\mathrm{Hg}$ under both field and laboratory exposures. ${ }^{5,7}$ A toxicokinetics trial that considers this eye component would elucidate more information about its high accumulation capacity. The presence of protective barriers in the brain and eyes is distinct from the case with internal organs, such as the liver, and would certainly have implications on $\mathrm{Hg}$ fate over time. 
1 The evaluation of $\mathrm{Hg}$ toxicokinetics in post-exposure periods is still poorly documented. The presence of BBB and BRB would probably limit the elimination of $\mathrm{Hg}$ from the brain and eyes, respectively. This was reported for Fe in rodents' brains, ${ }^{8}$ but no information is available for fish brains or eyes regarding $\mathrm{Hg}$. Again, both barriers would probably lead to distinct elimination patterns between barrierprotected and non-protected organs (e.g. the liver), but this hypothesis needs elucidation. Additionally, fish in their natural environment could easily move around areas with different contamination profiles, 10 making the assessment of $\mathrm{Hg}$ fate in their body after the cessation of exposure critical. Thus, following up the $\mathrm{Hg}$ levels in fish key organs during a depuration period will also provide a relevant indicator for an assessment of environmental health.

Several advances have been made elucidating the bioaccumula15 tion of $\mathrm{Hg}$ in fish, both from food and water sources. ${ }^{9-12} \mathrm{MeHg}$ has been preferentially addressed in relation to $\mathrm{iHg}$, probably based on the presumption of its higher toxicity, related with its rapid uptake and distribution. However, it was stated that different forms of mercury share the same toxic chemical entity and that toxicity depends mainly on differential bioavailability. ${ }^{13}$ It was also reported that $\mathrm{iHg}$ can display stronger acute effects on fish than organic forms can. ${ }^{1} \mathrm{iHg}$ has also been revealed to be more potent than $\mathrm{MeHg}$ in inhibiting glutamine synthetase activity in fish cortical astrocytes. ${ }^{14} \mathrm{iHg}$ compounds, such as mercuric chloride, can also act as a direct $\mathrm{BBB}$ toxicant, thus increasing its permeability in rodents. ${ }^{15}$ Moreover, iHg can occur as a product of $\mathrm{MeHg}$ demethylation in the intestine and in the brain, ${ }^{16}$ highlighting the relevance of investigating the toxicokinetics of $\mathrm{iHg}$ forms. The importance of such knowledge is consubstantiated by the fact that the majority of $\mathrm{Hg}$ in natural waters occurs in inorganic forms, while $\mathrm{MeHg}$ often contributes to less than $5 \%$ of the total $\mathrm{Hg}$ in water. ${ }^{17}$

Published data concerning the accumulation of $\mathrm{iHg}$ in the key tissues/organs of fish following waterborne exposure are still insufficient to understand its toxicokinetics. In particular, there is a lack of information on $\mathrm{iHg}$ disposition during the post-exposure period and its elimination pathways. Fish neurosensory structures, such as the eyes, need to be considered in order to mitigate the lack of scientific knowledge on their role in $\mathrm{iHg}$ uptake and accumulation. Hence, this study considers the uptake, distribution and accumulation of $\mathrm{iHg}$ in a novel combination of tissues/organs (gills, eye wall, lens, blood, liver, brain) of the white sea bream (Diplodus sargus) over 14 days of exposure. Afterward, a depuration period of 28 days was considered in order to evaluate the elimination of $\mathrm{iHg}$ in those tissues/organs, as well as bile's role in that process. Mercury enrich5 ment factors were calculated to evaluate tissue-/organ-specific affinity for $\mathrm{iHg}$. Moreover, the rate of $\mathrm{Hg}$ elimination was estimated in order to clarify the recovery of each tissue/organ. Fish were exposed to realistic waterborne $\mathrm{Hg}$ concentrations in order to produce reliable data for an assessment of environmental health.

\section{Experimental}

\subsection{Experimental set-up and tissues/organs sampling}

55 Juvenile white sea breams (Diplodus sargus) provided by an Aquaculture Research Station (IPMA - Olhão, Portugal), from the same cohort (weight: $146 \pm 14 \mathrm{~g}$; total length: $19 \pm 1 \mathrm{~cm}$ ), were used in the experiment. Fish were held in $300 \mathrm{~L}$ fibreglass tanks at an average initial density of $0.012 \mathrm{~kg} \mathrm{~L}^{-1}$, under a 10:14 light: dark photoperiod. Seawater was renewed daily (around $80 \%$ ) and the fish were fed once a day with a commercial dry food [standard $3 \mathrm{~mm}$ from Sorgal (Portugal)], 1-2 hours before water renewal. Total $\mathrm{Hg}$ levels in the food pellets were lower than $0.01 \mu \mathrm{g} \mathrm{g}^{-1}$. On the sampling days, the fish were not fed in the 12 hours preceding fish handling. Water temperature, salinity and $\mathrm{pH}$ were monitored daily throughout the experiment, varying as follows, respectively: $13.5 \pm 0.3{ }^{\circ} \mathrm{C}, 35$ \pm 2 and $7-8$.

Prior to $\mathrm{Hg}$ exposure, the fish were allowed to acclimatize to the experimental conditions and routines for two weeks. Eight fish were sacrificed at the beginning of the experiment and used as the initial reference group (time zero; T0) (Fig. 1).

In exposure tanks, $\mathrm{HgCl}_{2}$ (Sigma Aldrich) was added to the water in an aqueous solution in order to reach a final concentration of $2 \mu \mathrm{g} \mathrm{L}^{-1}$. Mercury chloride was added on a daily basis after water renewal (i.e. daily water recontamination) during the exposure period. This $\mathrm{iHg}$ level was established by considering previous studies in contaminated areas ${ }^{7,18}$ in order to mimic environmentally realistic conditions. The control fish were kept throughout the experiment in tanks filled with clean seawater. Fish well-being required permanent attention, in accordance with national and international guidelines for the protection of animal welfare.

The fish were exposed to $\mathrm{HgCl}_{2}$ for 1 (E1), 3 (E3), 7 (E7) and 14 (E14) days. Thereafter, the fish were transferred to clean water (post-exposure) and allowed to recover for 14 (PE14) and 28 days (PE28) (Fig. 1). In each sampling time, eight fish were sampled per condition $(n=8)$. The experiment had a total duration of 42 days. Immediately after collection, the fish were anesthetized, weighed, measured, and sacrificed by cervical transection. Blood was collected with heparinised Pasteur pipettes from the cardinal vein, and the gills, eyes, liver, brain and bile were removed. The gills were carefully washed with distilled water and the filaments carefully separated. The eyes were dissected to isolate the lens and the remaining components, hereafter collectively called "eye wall" to simplify, encompassing the eye wall (retina, sclera, cornea, ciliary body, etc.), chambers' contents (vitreous and aqueous humours), and other small structures. ${ }^{7}$ All the biological samples were stored at $-80{ }^{\circ} \mathrm{C}$ until used in further processing for $\mathrm{Hg}$ determinations.

During the exposure period (at days 1, 3, 7 and 14), water samples were collected in triplicate from the exposure and control tanks 24 hours after recontamination to quantify the total $\mathrm{Hg}(\mathrm{tHg})$ levels, in order to prove that the fish were subjected to the toxicant. The values of $\mathrm{tHg}$ in the exposure

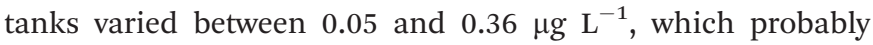
corresponds to the minimum exposure concentration. Levels of tHg in the control tanks were below the detection limit throughout the experiment $\left(0.1 \mathrm{ng} \mathrm{L}^{-1}\right)$. Identically, at days 28 and 42 (post-exposure period), both in the control and in the previously contaminated tanks, tHg was below the analytical detection limit. 


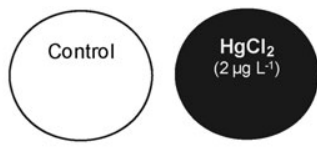

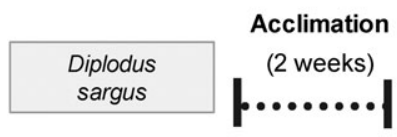
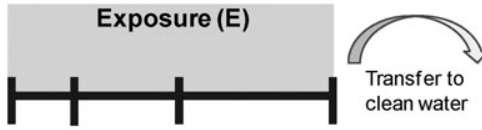

Post-exposure (PE) clean water

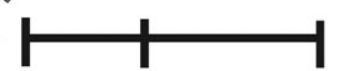

28

14

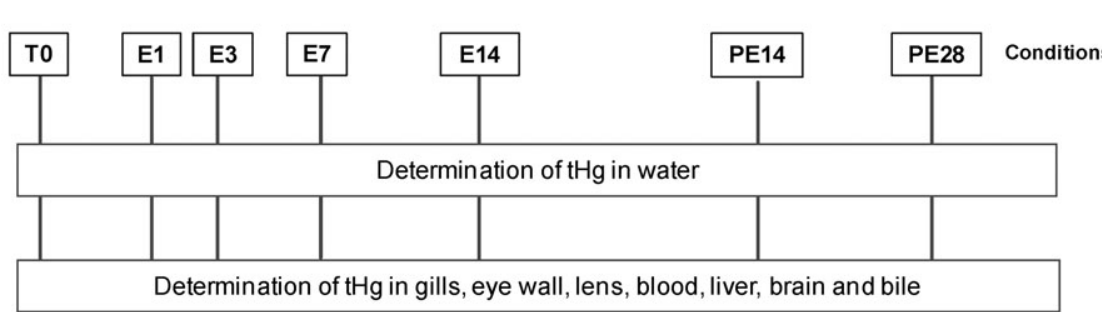

1

5

Fig. 1 Design of the experiment with white sea bream (Diplodus sargus), comprising $\mathrm{HgCl}_{2}$ exposure $\left(2 \mu \mathrm{g} \mathrm{L}{ }^{-1}\right)$. Prior to $\mathrm{Hg}$ exposure, the fish was allowed to acclimatize for 2 weeks (TO). The fish was exposed for 1, 3, 7 and 14 days (conditions E1, E3, E7 and E14, respectively). Thereafter, the fish was transferred to clean water and allowed to recover for 14 and 28 days (PE14 and PE28 conditions, respectively). In parallel, control groups were also considered.

\subsection{Analytical procedures}

The total dissolved mercury was determined following U.S. EPA method 1631. ${ }^{19}$ Briefly, water samples were preserved by the addition of $0.5 \% \mathrm{BrCl}$ until the analyses (less than one week after collection). The samples were then analyzed by coldvapour atomic fluorescence spectrometry (CV-AFS) with a PSA model Merlin 10.023 equipped with a detector PSA model 10.003 using $\mathrm{SnCl}_{2}$ reduction. BCR-579 reference material was used to control the accuracy of the procedure, and it was confirmed that the obtained values were consistent with the certified ones.

Gills, eye wall, lens, blood, liver, brain and bile samples were first lyophilised and homogenised. The samples were then analysed for $\mathrm{tHg}$ by atomic absorption spectrometry (AAS) with thermal decomposition, followed by gold amalgamation, using a mercury analyser (AMA) LECO 254. ${ }^{20}$ Certified reference materials (DORM-3, DOLT-4) were used to ensure the accuracy of the procedures, and it was confirmed that the obtained values were consistent with the certified ones.

In the current work, $\mathrm{tHg}$ levels in biological samples allowed interpretations on $\mathrm{iHg}$ toxicokinetics, based on the assumptions that fish were exposed to $\mathrm{iHg}$ and that no methylation has so far been reported to occur in fish.

\subsection{Data analysis}

Statistical software (Statistica 6.0) was used for the statistical analyses. All the data were first tested for normality (ShapiroWilk test) and homogeneity of the variance (Levene's test) to meet statistical demands. A one-way analysis of variance (ANOVA) was performed to compare tHg levels in the control and exposed fish for each experimental time. The comparison was performed for the six analysed tissues/organs (gills, eye wall, lens, blood, liver, brain) and the bile. One-way ANOVA was also used to compare the experimental times for tHg levels accumulated in the control and exposed fish. The Tukey test was applied for post hoc comparison. Differences between the means were considered significant when $p<0.05$.

The quotient of tHg levels (mean values) in the control and exposed and fish were calculated for each experimental time and for all the analysed biological matrices. That quotient corresponds to the $\mathrm{Hg}$ enrichment factor.

Spearman analysis was used to test the significance of correlations between all the analysed biological matrices for $\mathrm{Hg}$ levels. The significance of correlations between $\mathrm{Hg}$ levels (in the exposed fish) and time (in days) during the exposure period was also tested by Spearman analysis. Correlations were considered significant for $p<0.05$.

A crude estimation of the rate of $\mathrm{tHg}$ elimination per day $(k)$ was made for the data obtained in the post-exposure period, as

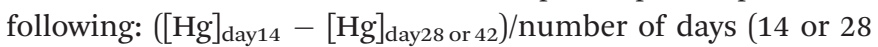
days). The elimination rate $(k)$ was expressed as $\mu \mathrm{g}$ of $\mathrm{Hg} \mathrm{g}^{-1}$ of tissue per day.

\section{Results}

No fish mortality was observed during the experiment. Though feeding was not strictly monitored, no alterations were perceptible during and after treatment on fish feeding-behaviour.

\subsection{Mercury levels in fish tissues/organs and bile}

Fig. 2 presents the variation of total $\mathrm{Hg}(\mathrm{tHg})$ in the gills, eye wall, lens, blood, liver, brain and bile of white sea bream exposed to inorganic $\mathrm{Hg}(\mathrm{iHg})\left(2 \mu \mathrm{g} \mathrm{L}^{-1}\right)$, as well as in the control fish. In the gills, tHg levels differed significantly between the control and exposed fish after the first day of exposure. Hence, tHg levels in the gills increased gradually and reached a maximum at E14, 

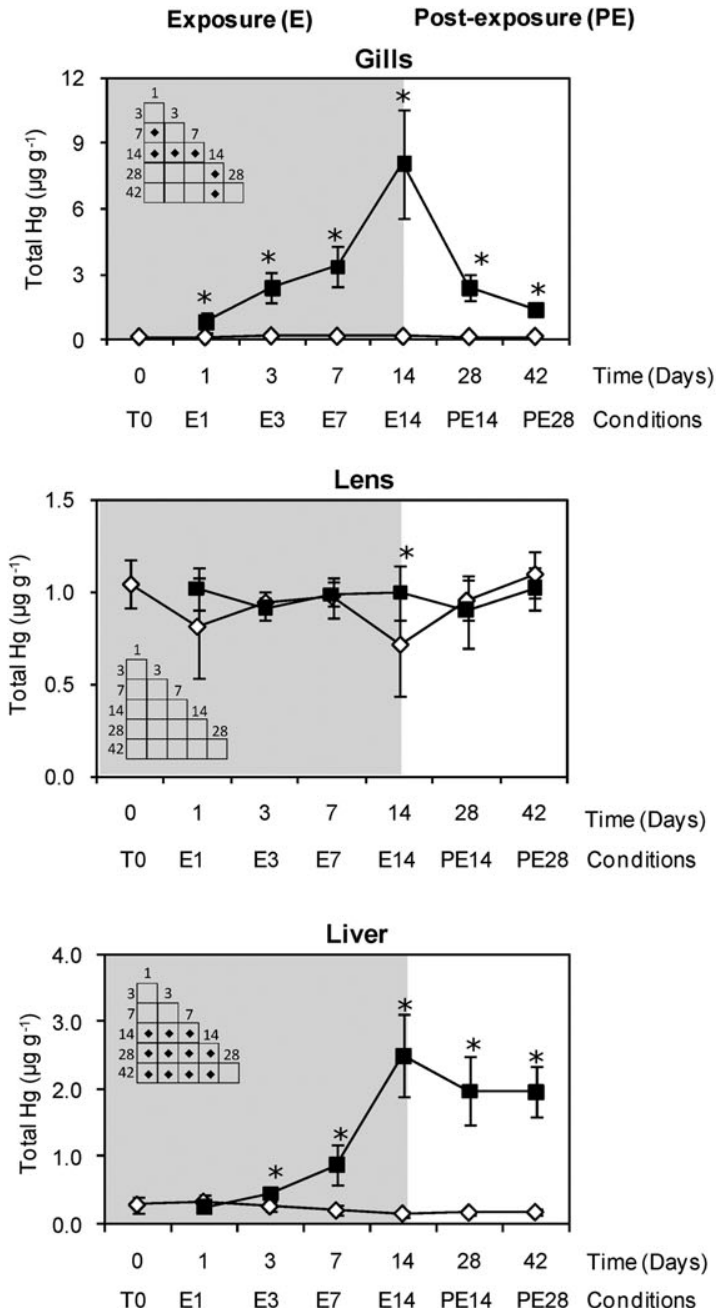

Bile

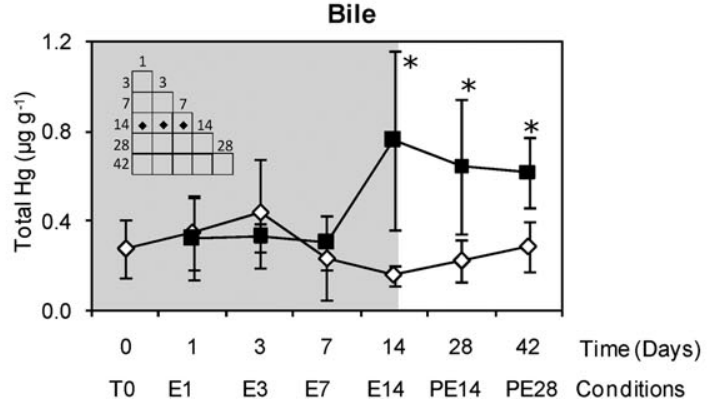

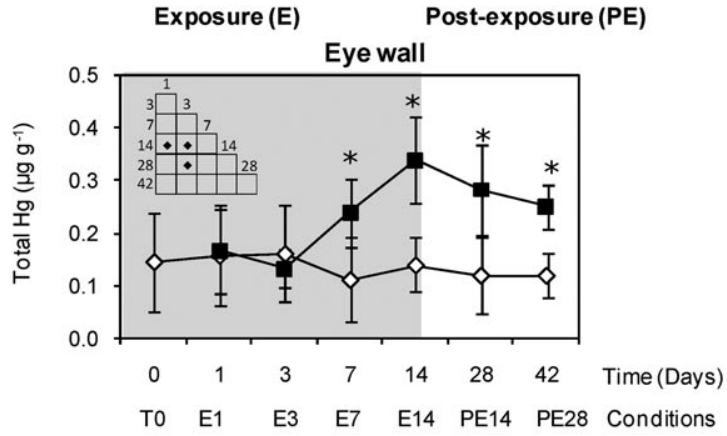
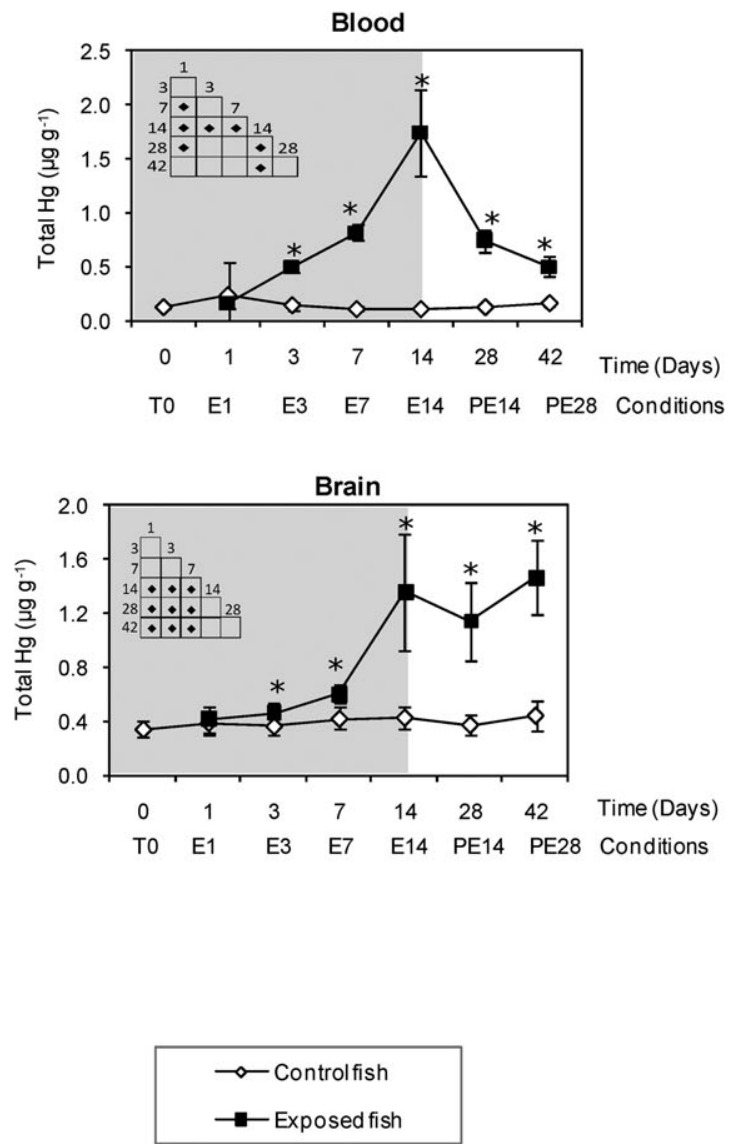

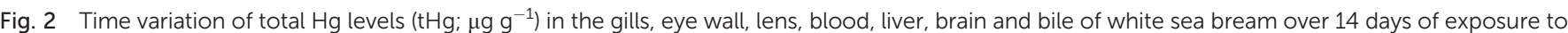

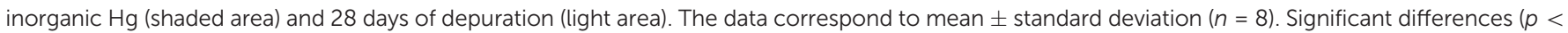

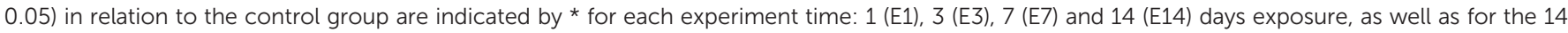

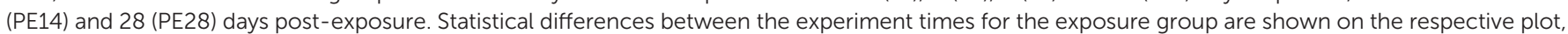
signalized by $\bullet$. tHg levels in the fish prior to the experiment (TO) are also indicated.

which presented significantly higher values than those recorded at days 1, 3 and 7. Moreover, tHg levels at E7 were significantly higher than the values at E1. In the post-exposure period (PE14 and PE28), tHg levels in the gills decreased significantly in relation to E14, but remained above the values in the control.
A similar variation pattern was found for the blood, liver and brain in the exposure and post-exposure periods, being characterized by significant differences between the control and exposed fish at the conditions: E3, E7, E14, PE14 and PE28 (Fig. 2). As observed for the gills, tHg levels in the blood, liver and brain reached a maximum at day 14 . Concentrations of $\mathrm{tHg}$ 
1 in the blood and liver decreased significantly in the postexposure period (PE14 and PE28), but remained always above the control values. In contrast, tHg levels in the brain were identical in conditions E14, PE14 and PE28. Similarly, tHg levels in the eye wall did not decrease significantly in the post-exposure period in relation to the last day of exposure (E14). During the exposure period, significant differences were found between $\mathrm{tHg}$ levels in the eye wall of the control and exposed fish at E7 and E14. Maximum levels of tHg in the eye wall were recorded at E14, as described for the remaining tissues. No statistical differences were found between tHg levels in the control and exposed fish for the lens, with the sole exception of E14. Moreover, tHg levels in the lens of exposed fish did not vary significantly during the experiment. Regarding the bile, tHg levels differed significantly between the control and exposed fish at E14, PE14 and PE28, while the values did not decrease significantly in the post-exposure period in relation to E14.

With the exception of the lens, tHg levels in the postexposure period never reached the levels found in the control fish (Fig. 2). In general, no temporal variations were found for tHg levels in the control fish.

The highest tHg levels accumulated throughout the experiment in the exposed fish were observed in the gills $\left(8.1 \mu \mathrm{g} \mathrm{g}^{-1}\right)$, followed by the liver $\left(2.5 \mu \mathrm{g} \mathrm{g}^{-1}\right)$, blood $\left(1.8 \mu \mathrm{g} \mathrm{g}^{-1}\right)$ and brain $\left(1.5 \mu \mathrm{g} \mathrm{g}^{-1}\right)$, and then by the lens $\left(1.0 \mu \mathrm{g} \mathrm{g}^{-1}\right)$, bile $\left(0.76 \mu \mathrm{g} \mathrm{g}^{-1}\right)$ and eye wall $\left(0.34 \mu \mathrm{g} \mathrm{g}^{-1}\right)$ (Fig. 2).

\subsection{Enrichment factors of mercury in fish tissues/organs}

The enrichment factors of $\mathrm{tHg}$ at each sampling time varied between the analysed tissues, as well as over time (Fig. 3). The gills exhibited the highest enrichment factors during the exposure period (1-14 days) (6-49), followed by the blood (1-16), liver (1-15), brain (1-3) and eye wall (1-2). In general, the enrichment factors increased with the exposure time (reaching the maximum values at day 14), followed by a decrease in the post-exposure phase. The gills, blood and liver showed an accentuated decrease of the enrichment factors between E14 and PE14, as following, respectively, from 49 to 16 ; from 16 to 6; from 15 to 11 . The enrichment factors of the gills and blood continued to drop between PE14 and PE42 (from 16 to 9 and from 6 to 3, respectively), while values in the liver remained the same in both post-exposure times. A different temporal pattern was recorded for the brain and eye wall in comparison with the previous biological matrices, since the enrichment factors were the same in E14, PE14 and PE28 conditions (always 3 for the brain and 2 for the eye wall). No substantial time-related changes were observed for the lens during both the experimental periods, with the enrichment factors staying around 1 .

\subsection{Relationships between mercury levels and exposure time}

In the exposed fish, tHg levels in all the biological matrices (except the lens) increased linearly with exposure time (Fig. 4). The slope of the relationship between tHg and time in the gills (0.535) was around three- and five-fold higher than those of the liver (0.174) and blood (0.117), respectively. The slopes of the relations of $\mathrm{tHg}$ in the brain (0.073), bile (0.033) and eye wall (0.015) vs. time were one order of magnitude lower than the previous ones. In contrast, no significant correlation was found between tHg levels in the lens and exposure time.

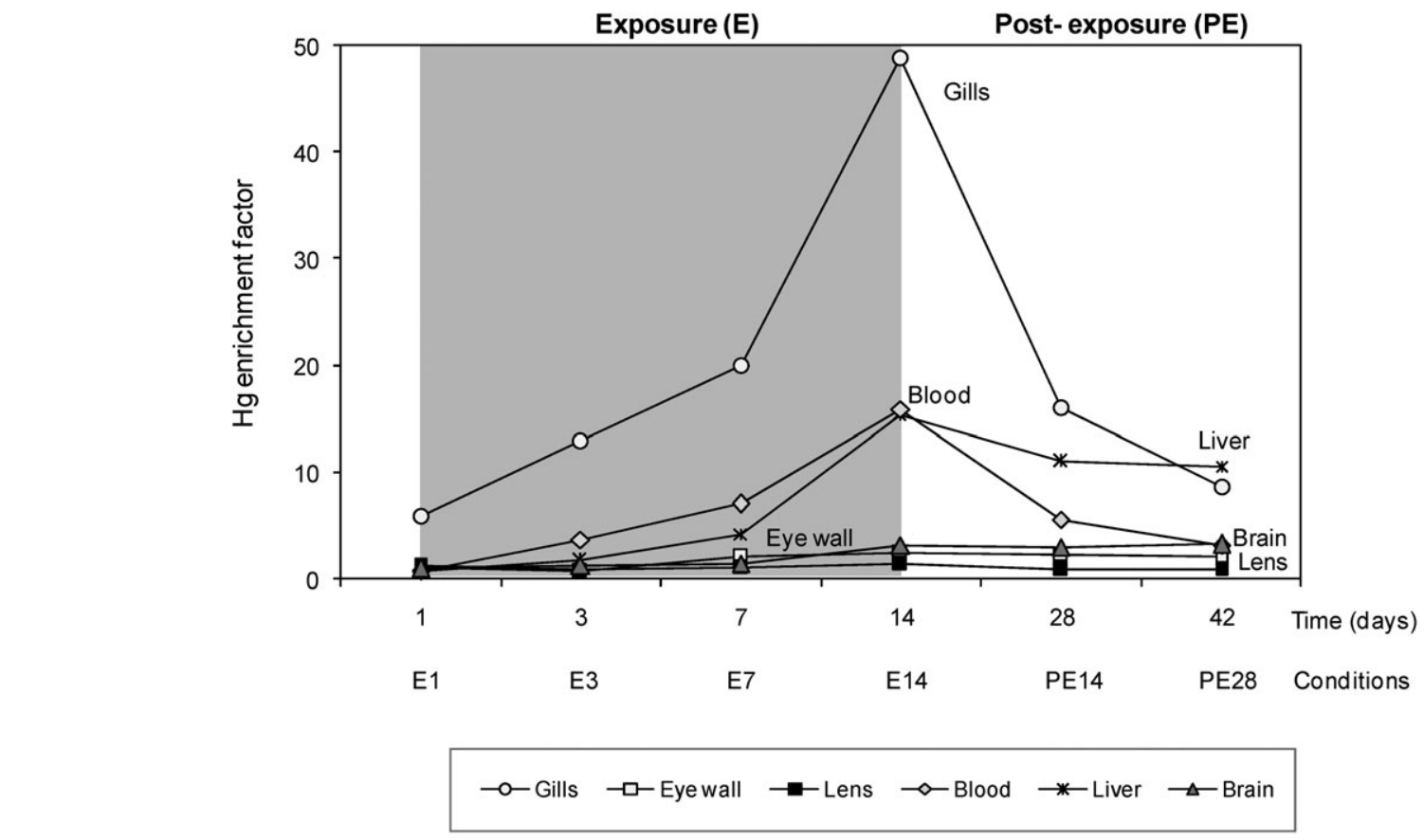

Fig. 3 Time variation of the enrichment factors calculated by the quotient between the mean of the total $\mathrm{Hg}$ (tHg) levels in the exposed and control fish. 

and mercury levels

Fig. 5 presents the significant correlations found between the analysed biological matrices for tHg levels after the Spearman analysis (note, the non-significant correlations are omitted). obtained in the post-exposure sampling days.

In the exposure period, the tHg levels recorded in the gills significantly correlated with the values found in the blood, liver and brain, while the levels in the liver highly correlated with those in the blood and brain. Additionally, tHg levels in blood significantly correlated with those in the brain.
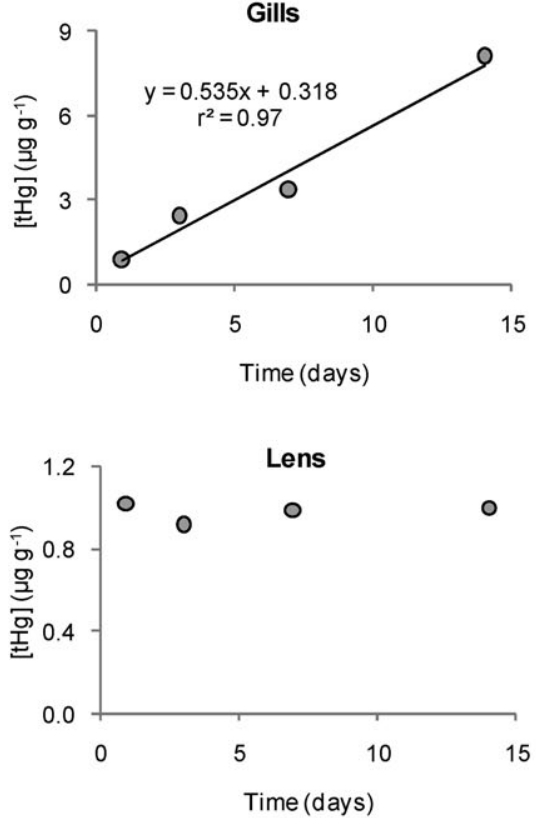

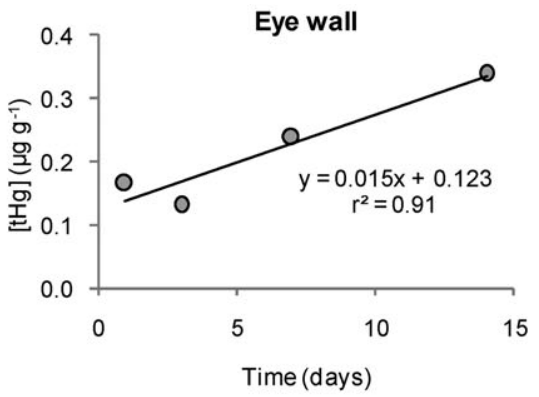

1
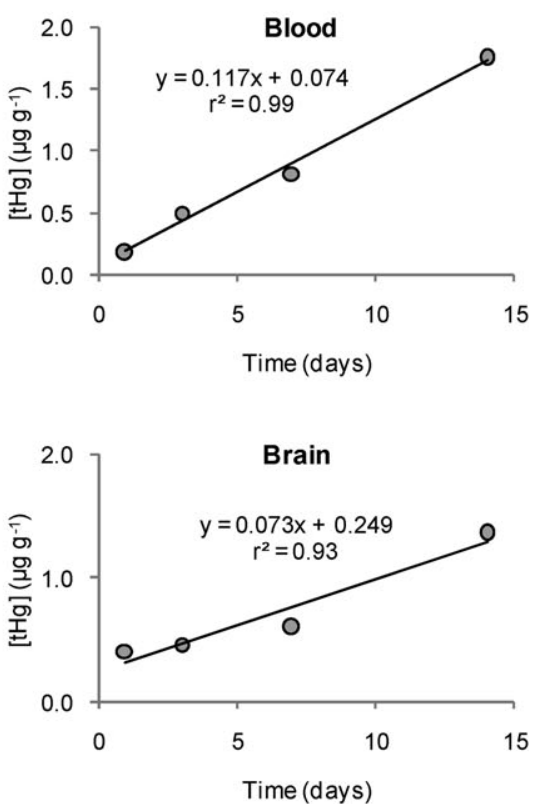

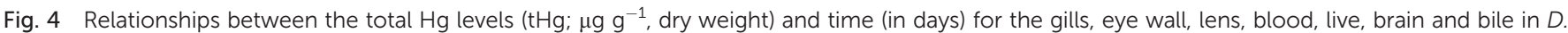
sargus exposed to $\mathrm{HgCl}_{2}$. Only mean $\mathrm{tHg}$ levels of he exposed fish and solely in the exposure period (๑) are plotted.

In the post-exposure phase, $\mathrm{tHg}$ levels did not significantly correlate among the analysed tissues. Interestingly, a sole exception was found for the brain vs. blood, with $\mathrm{tHg}$ levels in both tissues being negatively correlated.

\subsection{Rate of mercury tissue elimination}

At PE14, the rate of $\mathrm{iHg}$ elimination $(k)$ in the gills (0.40) was 20-, 10 - and 6-fold higher than those of the brain (0.02), liver $(0.04)$ and blood (0.07), respectively (Table 1$)$. The $k$ values estimated for the eye wall and lens were one order of magnitude lower than the previous ones $(0.004,0.008$, respectively). In general, 


\subsection{Mercury uptake, distribution and accumulation in key tissues}
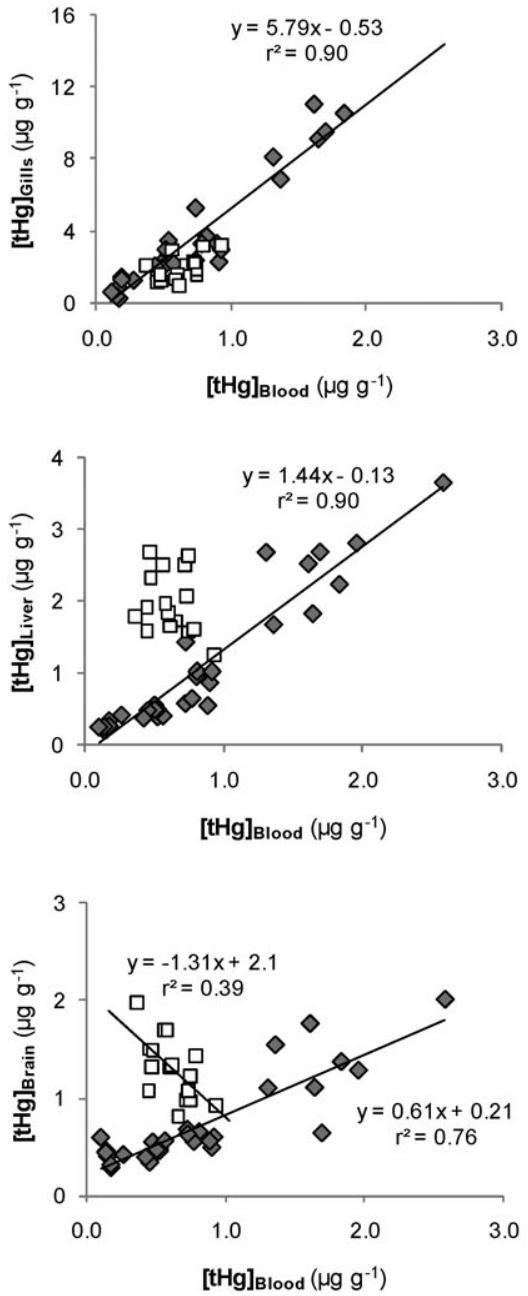
The exposure period $(\downarrow)$ and post-exposure period $(\square)$ are plotted separately.
The current results revealed that the gills accumulate $\mathrm{iHg}$ faster

Table 1 Estimated rate $(k)$ of inorganic mercury $(\mathrm{iHg})$ elimination for each tissue/organ during the post-exposure period (PE)

\begin{tabular}{|c|c|c|c|c|c|c|}
\hline \multirow[b]{2}{*}{ Condition } & \multicolumn{6}{|c|}{ Rate of iHg elimination $(k)\left(\mu \mathrm{g} \mathrm{Hg} \mathrm{\textrm {g } ^ { - 1 }}\right.$ per day) } \\
\hline & Gills & Eye wall & Lens & Blood & Liver & Brain \\
\hline 14 & 0.40 & 0.004 & 0.008 & 0.07 & 0.04 & 0.02 \\
\hline PE28 & 0.07 & 0.002 & -0.01 & 0.02 & 0.001 & -0.02 \\
\hline
\end{tabular}

the elimination of thg slowed down in the post period (i.e. in the 15-28 days of recovery) in all the biological matrices. In particular, the brain and lens presented a negligible rate of $\mathrm{iHg}$ elimination in that period.

\section{Discussion}

(within 1 day) than the remaining tissues when the fish are exposed to realistic levels of this $\mathrm{Hg}$ counterpart via the water.
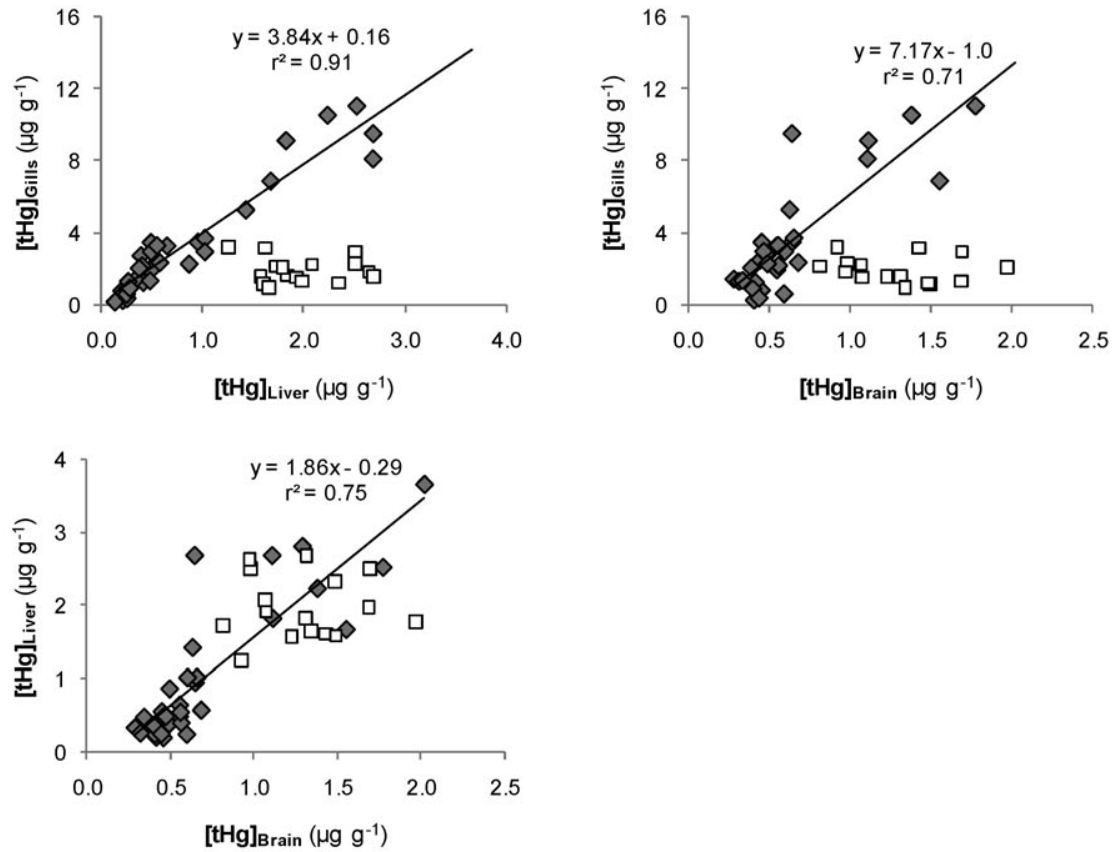

$$
\begin{aligned}
& \diamond \text { Exposure period } \\
& \text { वPost-exposure period }
\end{aligned}
$$

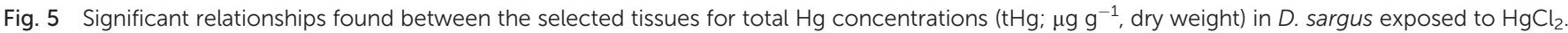

The high responsiveness of the gills was also corroborated by the maximum values of $\mathrm{Hg}$ enrichment factors being obtained during the exposure period. This is not surprising, since it is well established that the gills are the primary route for waterborne iHg to enter in to fish e.g. ref. 1 and 3. The preponderance of the gills as an uptake surface could explain the significant correlations obtained for tHg levels in the gills and liver in the exposure period, as well for the strong association of the gills with the blood and brain. In fact, the gills are in direct contact with water and suspended particles, and are thus a relevant interface with metal ions, ${ }^{21,22}$ including $\mathrm{iHg}^{3}{ }^{3}$ Several authors have claimed that $\mathrm{iHg}$ accumulates less than $\mathrm{MeHg}$, due to its lower lipophilicity e.g. ref. 23. However, current results suggest that $\mathrm{iHg}$ could also be rapidly taken up by the gills (i.e. significant differences from the control within 1 day of exposure). It is still unclear whether iHg absorption by the gills is through a physiologically regulated transport or by passive diffusion. It was previously suggested that iHg uptake involves a number of mechanisms, both active and passive, and that iHg binds strongly to the gills (e.g. to SH groups) ${ }^{24}$ Additionally, the accumulation of non-essential waterborne metals by the gills of 
1 freshwater fish is generally thought to occur when metals (like $\mathrm{Hg}$ ) are taken up inadvertently by transport processes designed for the essential cations (e.g. $\mathrm{Cd}^{2+}$ uptake instead of $\left.\mathrm{Ca}^{2+}\right) \cdot{ }^{24} \mathrm{Hg}$ entry into the gills might also be facilitated by the physiological gradient enhancement, due to the counter-current principle between the flows of water and blood outside and inside the gill structures. The present data on D. sargus confirmed that $\mathrm{iHg}$ taken up by gills enters the bloodstream, as indicated by the strong relationship found between $\mathrm{tHg}$ levels in the gills and in blood, which is in line with previous studies. ${ }^{12}$

Fish eyes are also in direct contact with the surrounding medium, but the significant increase of accumulated $\mathrm{Hg}$ in the eye wall was only noticed after 7 days of exposure. The temporal delay found between the gills and eye wall for $\mathrm{iHg}$ load 5 increases could be attributed to the distinct nature and physiology of the tissues. Despite the direct contact of fish eyes with water, this organ seems to be, to some extent, impervious to the dissolved $\mathrm{iHg}$, and thus is physiologically protected. The epidermal mucus secretions covering fish eyes could be the first line of defence against metals. ${ }^{25}$ Several mucus constituents of fish skin, such as the sialic acid and other glycoprotein components, may bind and immobilize $\mathrm{iHg}^{1}$ preventing its direct uptake by the eyes. iHg can also induce mucous secretion in the gills of various species of fish as a defensive mechanism, ${ }^{25}$ but data in sea bream suggest that its chemical composition is probably different from the eyes' mucus and does not efficiently entrap iHg at the gills' surface. Additionally, current data suggest that water is not the main vehicle of $\mathrm{iHg}$ entering fish eyes, suggesting the occurrence of an alternative pathway for $\mathrm{iHg}$ to reach the eye wall. Instead, iHg can be distributed through the blood to the eye wall and this seems to be the preferential uptake route. Such a distribution was previously observed for $\mathrm{MeHg}$ in zebrafish ${ }^{5}$ and was invoked to explain the $\mathrm{iHg}$ accumulation in wild fish. ${ }^{7}$

Indeed, blood is the main vehicle for mercury (re)distribution in the fish body (similar to other xenobiotics). Except for the eye wall, the blood can also transport substantial amounts of $\mathrm{iHg}$ to the liver and brain, which explains the common temporal pattern found between the two organs in the exposure 0 period. However, the liver accumulated higher $\mathrm{iHg}$ levels than the brain at E14 (mean values of $2.5 \mu \mathrm{g} \mathrm{g} \mathrm{g}^{-1}$ and $1.4 \mu \mathrm{g} \mathrm{g}^{-1}$, respectively) and showed greater enrichment factors. These differences could be attributed to the fact that $\mathrm{iHg}$ only reaches the brain after crossing the blood-brain barrier (BBB), while no physiological external barriers exist to protect the liver, as the blood directly contacts hepatocytes through the large gaps of sinusoidal capillaries that exist in the hepatic lobules. Moreover, the liver has a well-recognized detoxification function, being a preferential site of metals' accumulation in fish, ${ }^{26-28}$ including in response to $\mathrm{iHg}$ water exposure. ${ }^{29} \mathrm{iHg}$ in fish liver cells was reported to be mainly located in lysosomes and nuclei. ${ }^{30}$ Mercury levels in blood were highly correlated with values in the brain and liver, but the slope was almost two-fold higher in the liver $v s$. blood (1.44) than in the brain $v s$. blood (0.61), indicating a higher transference of iHg in the first case. Such a difference supports the previous hypothesis that higher
$\mathrm{iHg}$ levels in the liver may result from the absence of protective external barriers in opposition to what occurs for the brain.

As previously stated for the brain, also fish eyes are protected by an epithelial barrier (blood-retinal barrier - BRB). The current exposures of sea bream revealed that $\mathrm{iHg}$ could cross both $\mathrm{BBB}$ and $\mathrm{BRB}$ within a few days of exposure and under realistic contamination levels of $\mathrm{iHg}$ in water. In fact, $\mathrm{BBB}$ and BRB strictly regulate the transport of molecules from the bloodstream to the cells of the brain and retinas, but $\mathrm{Hg}$ chloride can penetrate both barriers via membrane carrier systems. ${ }^{5}$ Interestingly, the current data suggest that the BBB is more permeable to $\mathrm{iHg}$ than BRB. A temporal comparison of tHg levels in both organs allows us to conclude that $\mathrm{iHg}$ reached the brain more rapidly (within 3 days of exposure) than with the eye wall (took 7 days of exposure). The greater susceptibility of the brain to $\mathrm{iHg}$ exposure is also highlighted by the higher accumulated levels $\left(0.42-1.4 \mu \mathrm{g} \mathrm{g}^{-1}\right)$ relatively to in the eye wall $(0.17-$ $\left.0.34 \mu \mathrm{g} \mathrm{g}^{-1}\right)$ and also by the enhanced enrichment factors over the exposure period (1.1-3.2 for the brain and 0.82-2.4 for the eye wall). The higher permeability of BBB in comparison with $\mathrm{BRB}$ to $\mathrm{iHg}$ is also supported by the fact that significant correlations were found between tHg levels for the brain $v s$. blood, while no associations were obtained for the eye wall $v s$. blood. The fish eye wall comprises probably pseudo-isolated components of the eye, which provide to such structures a singular iHg toxicokinetics, as suggested by the absence of significant correlations between $\mathrm{tHg}$ levels in the eye wall and the other biological matrices. Tissue-specificities concerning the tHg load in fish were also found under field exposure, with values ranging from 0.11 to $0.61 \mu \mathrm{g} \mathrm{g}^{-1}$ in the brain and 0.05 to $0.30 \mu \mathrm{g} \mathrm{g}^{-1}$ in the eye wall. ${ }^{7}$ Since mercury accumulation by eye tissues has only recently been revealed, ${ }^{5,7}$ more research is still needed to clarify the higher permeability of $\mathrm{BBB}$ to $\mathrm{iHg}$ in comparison with BRB. Thiols and MTs cysteine-rich intracellular proteins are important ligands for $\mathrm{iHg}$ in the central nervous system $(\mathrm{CNS})^{31}$ and this could be related to the distinct accumulation capacity of the brain and eye wall.

$\mathrm{Hg}$ (II) could also reach the brain by axonal transport, as previously stated, ${ }^{9}$ but this pathway is probably less important than $\mathrm{iHg}$ transport through the BBB. The mechanism by which $\mathrm{iHg}$ could reach the brain is still a controversial issue. $\mathrm{iHg}$ appearance in the brain was previously attributed to organic $\mathrm{Hg}$ uptake and subsequent demethylation. The transport of $\mathrm{iHg}$ to the brain via the blood after demethylation in the liver (which has been well established) is another widely accepted hypothesis. Current data contributes to demystifying this controversy, revealing that $\mathrm{iHg}$ could reach the brain after waterborne exposure and via the bloodstream. iHg would probably pass the BBB by diffusion, which is contrary to the previous assumptions of Rouleau et al. (1999) ${ }^{9}$ who described BBB as impervious to iHg. Mercuric chloride can also act as a direct BBB toxicant, affecting its structure and thus increasing its permeability. ${ }^{15}$

The lens did not reflect iHg exposure by water within 14 days of exposure. In fact, $\mathrm{tHg}$ levels in the fish lens remained relatively unchanged over the exposure time, and similar levels were recurrently found between the control and exposed fish. 
1 These results are in line with our most recent data for $\mathrm{iHg}$ accumulation in the lens of wild fish. ${ }^{7}$ Apparently, the lens is unable to reflect different environmental availabilities related with seasonal changes, as well as within the timeframes currently considered for sea bream. The absence of significant tHg increases in the lens of the exposed fish could be related with the chemical form of this trace element. The lens were shown to preferentially accumulate $\mathrm{MeHg}$ (more than 96\% of total $\mathrm{Hg}$ ) over iHg under field exposure, presumably due to its high 10 protein nature. ${ }^{7}$ Korbas and co-authors $(2013)^{5}$ investigated the uptake and accumulation of $\mathrm{MeHg}$ in zebrafish larvae, and found the highest levels in the secondary lens fibres, underlying the lens epithelium. The lens is the site of particularly high protein production (namely, crystallins) and deposi5 tion. $^{32} \mathrm{MeHg}$ reaches the lens from the aqueous humours, since it has no direct blood supply. Thus, it is plausible that under $\mathrm{iHg}$ exposure, this chemical form does not cross from the surrounding aqueous humours to the lens, due to the very low chemical affinity.

20 The maximum levels of $\mathrm{Hg}$ accumulation were recorded after 14 days of exposure for all the target tissues/organs (except the lens). This led to the maximum enrichment factors at E14. The mean levels of $\mathrm{tHg}$ reached by the gills, blood, liver and brain at E14 $(8.1 \pm 0.17,1.8 \pm 0.11,2.5 \pm 0.16,1.4 \pm$ $0.43 \mu \mathrm{g} \mathrm{g}^{-1}$, respectively) were high considering that the fish were only exposed to realistic waterborne iHg levels $\left(2 \mu \mathrm{g} \mathrm{L} \mathrm{L}^{-1}\right.$ $\mathrm{HgCl}_{2}$ ). These tissues exhibited higher concentrations than those found in another estuarine species (Liza aurata) from an area severe contaminated by $\mathrm{Hg}$ (mean values around $0.10 \mu \mathrm{g} \mathrm{g}^{-1}$ in gills, $0.05 \mu \mathrm{g} \mathrm{g}^{-1}$ in blood, $1.0 \mu \mathrm{g} \mathrm{g}^{-1}$ in liver and $0.20 \mu \mathrm{g} \mathrm{g}^{-1}$ in brain). ${ }^{26}$ Under field exposure, the fish were subjected to both inorganic and organic $\mathrm{Hg}$ counterparts, as well as to different absorption pathways, i.e. contaminated water and food. Thus, the higher levels of tHg currently found in sea bream tissues/organs highlight the relevance of both $\mathrm{iHg}$ in its chemical form and water as a vehicle for $\mathrm{Hg}$ entry into the fish body.

Mercury is highly reactive with sulphydryl groups of proteins, forming covalent bonds with reduced glutathione (GSH)

40 and cysteine residues of proteins. GSH is the primary antioxidant and conjugating agent, and acts as the first line of defence against $\mathrm{Hg}$. GSH was previously determined in the gills, liver and brain of Liza aurata from Aveiro lagoon. ${ }^{33,34} \mathrm{~A}$ significant decrease in GSH was recorded in the liver and brain 45 of fish from the most contaminated area, suggesting the release of GSH-Hg conjugates, while no spatial changes were found for the gills.

\subsection{Fish recovery after the cessation of waterborne iHg exposure}

The post-exposure periods of 14 and 28 days allowed a significant decrease of $\mathrm{tHg}$ accumulation in the gills, followed by in the blood and liver, indicating that such tissues eliminate $\mathrm{iHg}$ within a few days in the absence of the compound in the water. The tissue elimination rates estimated for sea bream blood and liver are within the reported efflux-rate constant for the first depuration phase in whole sweetlips $\left[0.07 \mathrm{~d}^{-1}\right] \cdot{ }^{35}$ The values of $k$ estimated for the gills were higher than those of blood and liver, as well as higher than those previously presented for the whole body of sweetlips. ${ }^{35}$ The elimination of $\mathrm{iHg}$ is probably significantly promoted by the high cellular turnover of the gills.

The liver could also excrete iHg into the faeces, as a result of biliary secretion. ${ }^{36}$ However, tHg levels in the liver and bile did not vary concomitantly with exposure and post-exposure periods. Concentrations in the bile only increased significantly in the last exposure time (E14) and in both recovery times (PE14 and PE28). This temporal lag points to the involvement of other hepatic defence mechanisms (at E3 and E7), such as MTs and glutathione. The exhaustion of these detoxification strategies probably leads to the significant excretion of $\mathrm{iHg}$ through the bile.

Despite the significant reduction of accumulated $\mathrm{tHg}$ levels in the gills, blood and liver during the post-exposure period, the values did not reach baseline levels (i.e. the levels recorded in the control fish). Thus, more than 28 days are probably required for complete $\mathrm{iHg}$ elimination in those tissues, which is in line with previous estimations of iHg half-life $\left(t_{1 / 2}\right)$ in whole tilapia $[18.0 \text { days }]^{3}$ and in whole sweetlips [25.4 days] $]^{35}$ after waterborne exposure.

Mercury load in the eye wall and brain did not decrease in the post-exposure period, diverging from the temporal pattern previously described for the gills, blood and liver. In fact, no significant differences were found for $\mathrm{tHg}$ levels recorded in the eye wall and brain at E14 versus PE14 and E14 versus PE28, suggesting negligible $\mathrm{iHg}$ elimination within this timeframe. A similar finding was previously reported for zebra-sea bream brain exposed to $\mathrm{MeHg}$ in water ${ }^{4}$ and zebrafish (eyes and brain) subjected to several $\mathrm{Hg}$ counterparts. ${ }^{5}$ Mercury levels in sea bream brain negatively correlated with those in blood, indicating that $\mathrm{iHg}$ was not removed from the brain. This is a quite relevant finding regarding the $\mathrm{iHg}$ kinetics in brain, and indicates the low elimination ability within only 28 days recovery. Current data also highlight that the eye wall and brain are the final targets for $\mathrm{iHg}$, as previously suggested for tilapia, which accumulated significant levels of $\mathrm{Hg}$ (II) in the head at the end of 30 days of depuration. ${ }^{3}$ This is probably due to the high affinity of $\mathrm{iHg}$ to the cellular and molecular components of both tissues. Moreover, iHg that reaches both the eye wall and brain could only be removed via the blood, implying its passage through $\mathrm{BBB}$ and $\mathrm{BRB}$, respectively. It seems that both barriers can also limit the release of $\mathrm{iHg}$ in to the bloodstream and, thus, its elimination. The efflux of mercury from the brain has received very little attention, and even then, only regarding the $\mathrm{MeHg}$ form, while no data exists for the eye wall. The efflux of $\mathrm{MeHg}$ through the brain capillary endothelial cells of BBB has already been proven to occur in association with glutathione, ${ }^{37}$ as reported in other cell systems. ${ }^{23}$ Despite that $\mathrm{iHg}$ could cross BBB bi-directionally, $\mathrm{iHg}$ influx and efflux from the brain are probably unbalanced, leading inevitably to its accumulation in the brain over time, as previously described for Fe. ${ }^{8}$ It could also be hypothesized 
1 that an $\mathrm{iHg}$ redistribution between the eye wall and lens could lead to a decrease in the accumulated levels in the eye wall, as previously proposed for $\mathrm{MeHg}$ in a field study. ${ }^{7}$ However, current data do not support this hypothesis, since tHg levels in the lens did not vary significantly over the depuration time, and the iHg enrichment factors were around 1.0 for both PE14 and PE28.

The slow release of $\mathrm{iHg}$ from the eye wall and brain upon the cessation of exposure is an important aspect, considering their

10 main physiological roles. Regarding the fish eyes, there are several implications on an organism's health and survival that could be expected due to the presence of iHg; for instance, blindness of fish has been reported after $\mathrm{iHg}$ exposure, ${ }^{38}$ as well as visual deficits observed, ${ }^{39}$ and disorganized retinas, abnor5 mal pigment distribution, and invasive blood sinuses in the eyes of medaka embryos exposed to $\mathrm{MeHg} .{ }^{40}$

Neurodegeneration was previously associated with the presence of $\mathrm{iHg}$ in the brain, ${ }^{31}$ and $\mathrm{iHg}$ has also been shown to be responsible for the inhibition of crucial molecular mechanisms, such as the thioredoxin system. ${ }^{4}$ Oxidative stress in fish brains has been attributed to the accumulation of inorganic $\mathrm{Hg}$ and organic $\mathrm{Hg}$ species under field exposure, ${ }^{41}$ and other neurotoxic effects, such as the reduction of neural monoamine oxidase activity and astrocyte proliferation, were also attributed to $\mathrm{iHg}$ e.g. ref. 42 , as well as behavioural impairments. ${ }^{43}$

The time retention of $\mathrm{iHg}$ in the fish eye wall and brain, and/ or the delayed efflux, is toxicologically relevant and should be taken into account when studying the health risk of wildlife exposed to iHg. Furthermore, the current findings can also be considered informative, on an extrapolation basis, to predict the risk of human exposure to $\mathrm{iHg}$.

\subsection{Contributions to the design of strategies for environmental health assessment using fish}

Understanding the $\mathrm{iHg}$ toxicokinetics is of utmost importance in the choice of the fishes' tissue/organs that could better reflect waterborne field contamination. Under this context, the short- and long-term exposures of fish to iHg need to be considered. According to current data relating to sea bream, the gills can be proposed as the most adequate tissue/organ to reflect short-term exposure to realistic levels of iHg. This conclusion is provided by two pieces of evidence: (i) the gills of exposed fish accumulated significantly higher levels of $\mathrm{iHg}$ in 1 day of exposure, compared to the control specimens; and (ii) the gills exhibited accumulation enrichment factors higher than 1 over the entire exposure period (14 days). Regarding the long-term exposure of fish to iHg, a more "refractory" tissue/organ seems to be more appropriate to more faithfully reflect water contamination. The brain and eye wall are considered the most appropriate "refractory" tissues/ organs in relation with $\mathrm{iHg}$ exposure, based on their slow elimination capacity. In fact, no significant elimination of $\mathrm{iHg}$ was detected in the brain and eye wall within 28 days of fish depuration. The $\mathrm{iHg}$ stability in those tissues/organs is particularly important when fish are considered in the assessment of aquatic contamination, due to its mobility. Such outcomes need to be considered in order to minimize the occurrence of false positive or negative results in environmental risk assessment.

\section{Conclusions}

These main findings were provided by the exposure of fish to realistic levels of waterborne $\mathrm{iHg}$ (within 14 days) followed by a depuration period of 28 days:

- The gills accumulated $\mathrm{iHg}$ faster than the eye wall, blood, liver and brain, reaching also the highest accumulation levels after exposure. In contrast, the lens was not able to accumulate iHg within this exposure timeframe;

- The physiological protection provided by BRB and BBB seems to be the reason for the lower $\mathrm{iHg}$ accumulation in the eye wall and brain, respectively, though the BBB was shown to be more permeable to iHg;

- 28 days of depuration were not enough to ensure the total elimination of $\mathrm{iHg}$ from any of the tissues, though biliary excretion was shown to be involved in $\mathrm{iHg}$ elimination during post-exposure. Moreover, the eye wall and brain were unable to achieve a significant elimination of iHg;

- The slow elimination of $\mathrm{iHg}$ in the eye wall and brain could represent a risk for wild populations of fish. These body compartments seem to be particularly informative of $\mathrm{iHg}$ water contamination under long-term exposure, whereas the gills could faithfully reflect short-term exposure.

\section{Ethical statement}

This study was conducted in accordance with the EU Directive 2010/63/EU on the protection of animals used for scientific purposes, under the supervision of a team member (Mário Pacheco) authorized by the competent authorities.

\section{Conflicts of interest}

There are no conflicts of interest in this work.

\section{Acknowledgements}

Patrícia Pereira (SFRH/BPD/69563/2010) and Joana Raimundo (SFRH/BPD/91498/2012) benefit from Post-doctoral grants supported by "Fundação para a Ciência e a Tecnologia" (FCT). This work has been supported by the Research project financed by FCT PTDC/AAG-REC/2488/2012 (NEUTOXMER - Neurotoxicity of mercury in fish and association with morphofunctional brain alterations and behavior shifts), as well as by the Centre for Environmental and Marine Studies (CESAM). Authors are also grateful to Ana Margarida Gamboa, Fátima Brandão and Pedro Brito for the support in the laboratory work, as well as to Mário Mil-Homens for his assistance with Adobe illustrator. 


\section{References}

1 C. A. Oliveira Ribeiro, J. R. D. Guimarães and W. C. Pfeiffer, Ecotoxicol. Environ. Saf., 1996, 34, 109.

2 C. A. Oliveira Ribeiro, E. Pelletier, W. C. Pfeiffer and C. Rouleau, Environ. Res., Sect. A, 2000, 83, 286.

3 R. Wang, M.-H. Wong and W.-X. Wang, Environ. Pollut., 2010, 158, 2694.

4 V. Branco, J. Canário, A. Holmgren and C. Carvalho, Toxicol. Appl. Pharmacol., 2011, 51, 95.

5 M. Korbas, B. Lai, S. Vogt, S.-C. Gleber, C. Karunakaran, I. J. Pickering, P. H. Krone and G. N. George, ACS Chem. Biol., 2013, 8, 2256.

6 R. A. Yokel, NeuroMol. Med., 2009, 11, 297.

7 P. Pereira, J. Raimundo, O. Araújo, J. Canário, A. Almeida and M. Pacheco, Sci. Total Environ., 2014, 494-495, 290.

8 J.-H. Chen, N. Singh, H. Taya and T. Walczyk, Metallomics, 2014, 6, 1417.

9 C. Rouleau, K. Borg-Neczak, J. Gottofrey and H. Tjälve, Environ. Sci. Technol., 1999, 33, 3384.

10 R. P. Mason, J.-M. Laporte and S. Andres, Arch. Environ. Contam. Toxicol., 2000, 38, 283.

11 C. A. Oliveira Ribeiro, C. Rouleau, E. Pelletier, C. Audet and H. Tjalve, Environ. Sci. Technol., 1999, 33, 902.

12 J. J. Leaner and R. P. Mason, Environ. Toxicol. Chem., 2004, 23, 2138.

13 S. De Flora, C. Bennicelli and M. Bagnasco, Mutat. Res., 1994, 317, 57.

14 J. W. Allen, L. A. Mutkus and M. Aschner, Brain Res., 2001, 891, 148.

15 W. Zheng, M. Aschner and J. F. Ghersi-Egea, Toxicol. Appl. Pharmacol., 2003, 192, 1.

16 M. Korbas, P. H. Krone, I. J. Pickering and G. N. George, J. Biol. Inorg. Chem., 2010, 15, 1137.

17 C. J. Watras, Sci. Total Environ., 1998, 219, 183-208.

18 P. Pereira, J. Raimundo, J. Canário, A. Almeida and M. Pacheco, Mar. Pollut. Bull., 2013, 77, 375.

19 U. S. EPA. Method 1630: Mercury in Water by Oxidation, Purge and Trap, and Cold Vapor Atomic Fluorescence Spectrometry, 2002, Washington, United States, Environmental Protection Agency.

20 C. T. Costley, K. F. Mossop, J. R. Dean, L. M. Garden, J. Marshall and J. Carrol, Anal. Chim. Acta, 2000, 405, 179.
21 C. Fernandes, A. Fontaínhas-Fernandes, F. Peixoto and M. A. Salgado, Ecotoxicol. Environ. Saf., 2007, 66, 426.

22 P. Pereira, H. de Pablo, C. Vale and M. Pacheco, Mar. Environ. Res., 2010, 69, 53.

23 G. P. Guzzi and C. A. M. Porta, Toxicology, 2008, 244, 1.

24 J. Klinck, M. Dunbar, S. Brown, J. Nichols, A. Winter, C. Hughes and R. C. Playle, Aquat. Toxicol., 2005, 72, 161.

25 W. E. Coello and M. A. Q. Khan, Arch. Environ. Contam. Toxicol., 1996, 30, 319.

26 C. L. Mieiro, M. Pacheco, M. E. Pereira and A. C. Duarte, J. Environ. Monit., 2009, 11, 1004.

27 P. Pereira, H. de Pablo, M. Pacheco and C. Vale, Ecotoxicol. Environ. Saf., 2010, 73, 805.

28 J. Raimundo, C. Vale, M. Caetano, B. Anes, E. Giacomello and G. Menezes, Deep Sea Res., Part II, 2013, 98, 137.

29 A. C. Elia, R. Galarini, M. I. Taticchi, A. J. M. Dörr and L. Mantilacci, Ecotoxicol. Environ. Saf., 2003, 55, 162.

30 E. Baatrup, M. G. Nielsen and G. Danscher, Ecotoxicol. Environ. Saf., 1986, 12, 267.

31 M. Farina, D. S. Avila, J. B. T. Rocha and M. Aschner, Neurochem. Int., 2013, 62, 575.

32 M. Korbas, P. H. Krone, I. J. Pickering and G. N. George, J. Biol. Inorg. Chem., 2010, 15, 1137.

33 C. L. Mieiro, I. Ahmad, M. E. Pereira, A. C. Duarte and M. Pacheco, Ecotoxicology, 2010, 19, 1034.

34 C. L. Mieiro, A. C. Duarte, M. E. Pereira and M. Pacheco, Ecotoxicol. Environ. Saf., 2011, 74, 2225.

35 W.-X. Wang and R. S. K. Wong, Mar. Ecol.: Prog. Ser., 2003, 261, 257.

36 R. K. Zalups, J. Toxicol. Environ. Health, Part A, 1998, 53, 615.

37 L. E. Kerper, E. M. Mokrzan, T. W. Clarkson and N. Ballatori, Toxicol. Appl. Pharmacol., 1996, 141, 526.

38 A. K. Panigrahi and B. N. Misra, Arch. Toxicol., 1980, 44, 269.

39 C. W. Hawryshyn, W. C. Mackay and T. H. Nilsson, Can. J. Zool., 1982, 60, 3127.

40 N. A. Dial, Teratology, 1978, 17, 83.

41 C. L. Mieiro, M. E. Pereira, A. C. Duarte and M. Pacheco, Aquat. Toxicol., 2011, 103, 233.

42 M. H. G. Berntessen, A. Aatland and R. D. Handy, Aquat. Toxicol., 2003, 65, 55.

43 L. R. Vieira, C. Gravato, A. M. V. M. Soares, F. Morgado and L. Guilhermino, Chemosphere, 2009, 76, 1416. 